\title{
Biobanking: shifting the analogy from consent to surrogacy
}

\author{
Ann M. Mongoven, $\mathrm{PhD}, \mathrm{MPH}^{1}$ and Stephanie Solomon, $\mathrm{PhD}^{2}$
}

\begin{abstract}
INTRODUCTION
Biobanks of various kinds are proliferating. Particularly noteworthy is the expanding development of highly institutionalized, broad population-based biobanks that collect and store blood or tissue to provide samples to the diverse scientific research community. Both the research potential and the ethical challenges of biobanks are attracting deserved public attention. The practice of using banked tissue samples in research without the knowledge of donors has become widely criticized, popularized by the best-selling book about Henrietta Lacks. ${ }^{1}$ (We use the problematic conventional term "donors" without regard to intent, to describe those who provide tissue to biobanks.) People have interests in how their banked tissue is used, even if their tissue is "de-identified": interests in the protection of privacy and confidentiality, and interests in supporting research consonant with their values while avoiding participation in research that contravenes them. ${ }^{2}$ A coherent, consistent, and cohesive approach to the ethics of biobanking is needed.

The prevailing ethical response has been to incorporate formal "informed consent" procedures into biobanking practice. Although well intended, this development is based on a flawed analogy - between the provision of a sample to a biobank and other forms of research participation. This analogy deserves to be critically examined, with both its insights and distortions explicitly highlighted. Since biobanking is a unique endeavor compared to other research, multiple analogies may be necessary to "think through" biobanking ethics.

In this commentary, we (i) call attention to the fact that current ethical conversation and regulatory approaches presume a straightforward analogy between biobanking and other forms of nontherapeutic research, without acknowledging that analogical reasoning is at play, (ii) elaborate the conceptual inadequacies of the presumed analogy between research and biobanking, particularly drawbacks of the informed consent model, (iii) invite a broadening of analytic scope to include other possible analogies, specifically considering an analogy between biobanking and clinical surrogacy, (iv) propose several ways that the surrogacy analogy better fits aspects of biobanking than the informed consent model, (v) suggest that multiple ethical analogies should illuminate the development of regulatory oversight.
\end{abstract}

\section{THE INCOHERENCE OF CONSENT}

Biobanking does share some significant ethical features with other nontherapeutic research: the goal of collective benefits, the intrinsic conflict of interest that goal generates regarding the necessary instrumental use of human subjects, and the need for ethical protections of human subjects to ensure they are treated as "ends in themselves" and not merely means to an end. The positive intent of regulatory frameworks that treat biobanking as research is to bring biobank participants under the umbrella of such protections. Those frameworks include the Department of Health and Human Services Office of Research Protection's definition of biobanking as an act of research. As a result, biobanks have been both subjected to institutional review board (IRB) oversight and required to meet concomitant requirements for consent and reporting. Proposed revisions to Common Rule $45 \mathrm{Cfr} 46$ would reaffirm the equation of biobanking with research while modifying review processes by perceived risk.

However, there are also fundamental disanalogies between biobanking and nontherapeutic research. An ethically central feature of informed consent for research is the opportunity for the research recruit to weigh potential risks and benefits of participation in a specific study. But that feature is not applicable to biobanking. The potential risks and benefits of the biobank's undetermined future research cannot be speculated. Biobanks do pose some risks, both to the informational security of donors and to the dignity of donors whose values may alternatively support or contraindicate certain forms of research. Significantly, however, these are not the risks of the actual future research.

This distinction leads to four other major differences between informed consent for research and informed consent for biobanking: (i) the difference between participating in an activity versus participating in an institution; (ii) the difference between one-time versus ongoing decision-making; (iii) the difference between unidirectional versus multidirectional information flows; and (iv) the difference between active versus detached research participation.

(i) In other forms of nontherapeutic research, the participant consents to participate in a specific activity-for example, a clinical drug trial. But in biobanking, the participant "consents" to contribute to an institution, the biobank. The biobank

Both authors contributed equally to the conceptual development of this project.

${ }^{1}$ Center for Ethics and Humanities in the Life Sciences and Department of Pediatrics and Human Development, College of Human Medicine, Michigan State University,

East Lansing, Michigan, USA; ${ }^{2}$ Albert Gnaegi Center for Health Care Ethics, Saint Louis University, Salus Center, St. Louis, Missouri, USA. Correspondence: Ann M. Mongoven

(ann.mongoven@ht.msu.edu) 
is a platform that supports research activities but is not itself a research activity.

(ii) The informed consent model envisions a one-time consent process. But this model is problematic for biobanking because the risks and benefits of research as well as of biobanking itself may change over time. Information provided at the time of donation cannot reflect this evolving environment. (At first glance, this could also be said of the traditional research context, in which institutional review boards require feedback to participants over time when it could affect their choice to participate. But in the traditional research context, this is seen as a rare extenuating circumstance, such as when medical advances show that a particular study drug works very well, or when standard of care changes. Even in longitudinal studies, which may last a long time, original research questions establishing consistent methods for data collection and management provide a framework for assessing risks and benefits. On the other hand, with biobanking, the changing environment of risks and benefits should be assumed, since biobanks by definition function for a long period of time.) Because biobanks store samples until researchers request them, there is an inevitable time lag between the time of "consent" and the actual research that could span from months to lifetimes. The time lag causes an unavoidable information gap about the future research. As a result, biobank consent documents use vague language regarding risks and benefits, confidentiality assurances, and utility of samples. These factors are dependent upon the state of technology, science, and the legal system when the samples are ultimately used, not when they are collected.

For example, although current technology does not allow tracking of research samples after they are distributed to researchers (currently biobanks require self-reporting), technological advances may make tracking possible in the future. Likewise, fully sequenced DNA is generally considered deidentified by current regulations because without identified DNA for comparison no reidentification can occur. But with the advances and increasing coordination of DNA technology, electronic medical records, and the like, isolated DNA samples may become much more easily identified in the future.

(iii) The informed consent model presumes a unidirectional information flow: the knowledgeable research staff provides information about the research, translated for lay-accessibility, to the research recruit so the recruit can decide whether to participate. In biobanking, that presumption is doubly problematic. The research staff lacks specific information to provide about the risks and benefits of future research. That lack implies the ethical importance of understanding recruits' general goals for participation, but the informed consent model does not require solicitation of such information.

(iv) Because biobanks function as intermediaries (both physical and informational) between donors and the ultimate research on their samples, donors are detached from the research in ways that are dissimilar from other research contexts. This detachment poses psychological as well as practical challenges to informed consent.

\section{EFFORTS TO ADAPT INFORMED CONSENT TO BIOBANKING}

Efforts to adapt informed consent to biobanking have taken two forms: "blanket" and "tiered" consent. Blanket consent gives broad leeway for the biobank to use the sample in future research that potentially advances medicine or public health. Some critics argue that most blanket consent processes provide only a superficial veneer of ethical legitimacy because they are too vague to be meaningful. ${ }^{4}$ Even utter transparency ("all risks and benefits of future research with your sample are unpredictable") would fail to fully solve problems with blanket consent. Transparency does not address the problem of physical and temporal detachment between donors and the future research uses of their samples, which may give the illusion of insulation from risk. Moreover, blanket consent is designed to be routinely instituted. Everyone who is treated in an institution, or whose newborn is screened for metabolic diseases, etc., is asked to consider blanket consent. When blanket consent becomes "just a routine request," its recruits may assume risks are minimal regardless of the specific wording of consent documents.

Some critics of blanket consent alternatively propose "tiered" consent processes. ${ }^{5}$ Tiered consent presents a list of predetermined options and limitations governing the future use of samples, allowing recruits to constrain their donation to advance more narrowly defined research goals than blanket consent or to avoid participation in research that violates their conscience. But tiered consent is simultaneously misleading and burdensome. It is misleading because there is no way of knowing whether the specific dictates will cohere with real future research options and real future values of the recruit. It is burdensome because its implementation may require a complex administrative and technological infrastructure unavailable to many biobanks.

Some commentators argue that computer technology enables meaningful and practicable tiered-consent options. They propose that creation of ongoing interactive virtual interfaces between biobanks and donors could allow donors to continuously revisit desired constraints on their sample use, or even to consent study by study. Considerable experimentation with such programs is under way. For example, Sharon Kardia and colleagues at the University of Michigan are working with informatics firm Private Access to adapt interactive computer models for clinical trial recruitment to biobanking consent processes (www.explorebiobanking.org). Although worthwhile to explore and evaluate, these programs ultimately shift the moral burden of time and information gaps between donation and use from researchers to potential donors. Although this may not be problematic for some populations heavily invested in research on particular problems (such as cohesive groups focused on particular diseases), most participants in biobanks are not so vested. They may have neither the time nor the interest for such continuous monitoring of their consent. Although proposed to counteract the detachment of biobank donors, such systems may fail because of that detachment. Moreover, banked samples will "outlive" their donors. Virtual systems for donors do not 
address questions of when, if ever, research beyond participant lifetime is consistent with respect for the deceased person. A recent empirical study suggests that potential biobank recruits recognize the administrative burden of tiered consent both on their side and on the biobanks.. ${ }^{6}$ S.B. Trinidad et al. move from empirical evidence of mixed participant support for multiple consent models to an argument for "respectful engagement" between participants and researchers, considering risk and benefits together through a stewardship governance model. ${ }^{7}$ In short, individualized tiered consent may be a concept that is elegant in theory but difficult to translate to effective practice.

Because the specific goals, risks, and benefits of future research are not known at the time of tissue donation, donors are not really "informed." Because decisions regarding the specific use of samples ultimately will be made by the biobank, donors are not really providing "consent." Highlighting the disanalogies between biobanking and other research contexts leads to stark ethical choices. The impossibility of true informed consent in the biobanking context could be acknowledged, with potentially beneficial research either forgone to protect donors from participation without informed consent or conducted under waivers from the obligation to seek consent that sacrifice the interests of individual donors for perceived social good. Or charades of consent could continue, posing dangers not only to biobank donors but also to the concept of informed consent itself. Its forced use in an inappropriate domain might erode its legitimate power in areas of biomedicine where the concept both makes sense and can be implemented.

\section{FROM CONSENT TO SURROGACY}

Such stark choices can be avoided by admitting that informed consent for research is an analogical model, and not the only one, that can be applied to biobanking. What potential biobank donors genuinely can be asked for is not consent for research but rather permission for the biobank to make future research decisions on their behalf. Such permission is based more on trust of those who will use the resource than on necessarily limited information about how the resource will be used.

Some have argued that informed consent processes for biobanking are important symbols of trust even though true informed consent is epistemically impossible. ${ }^{8}$ We believe it is more defensible to adopt ethical frameworks that explicitly acknowledge the limitations on informed consent in biobanking. These frameworks should explain the intrinsic limits on information and control available to biobank donors. They should explain how a biobank could become, or fail to become, trustworthy-and conversely how donors could make appropriately trusting or inappropriately trusting decisions to participate in biobanks.

The overconflation of biobanking and research, and corresponding disanalogies between the informed consent model and biobanking contexts, have resulted in inadequate attention to the true vulnerability of biobank donors. We suggest the value of exploring other analogies that shed light on the relationship between biobanks and their donors. Contemporary clinical medicine offers one such model: the designation of surrogate decision makers for incapacitated patients, informed by advance directives expressing patients' basic values. The quandary of biobank recruits, i.e., trying to imagine their interests in future unknowable research scenarios, is epistemically akin to that of present-competent patients trying to imagine their interests in future unknowable medical scenarios-although the risk of "incapacitation" for biobank donors is temporal and institutional rather than cognitive. Ideally, the designation of an appropriate surrogate by a patient is characterized by familiarity, trust, and a relationship through time. Each of these facets has a direct ethical analogue in responsible biobanking.

The first responsibility of a surrogate is to become familiar with patient values that should influence future surrogate decisions. Instead of merely providing vague information to recruits-the informed consent model-biobanks must solicit information from recruits. In the surrogacy model, familiarity with patient values is initially acquired from an advanced directive, and from conversations about the advance directive between patients and their designated surrogates. Implementation of advance directives has changed over the past generation. Both overly broad approaches ("no heroics") and overly narrow approaches ("if A happens do B") failed to be interpretable by surrogates in unimagined medical circumstances that actually arose. ${ }^{9}$ Significantly, those failed approaches directly parallel blanket and tiered consent in biobanking. In clinical medicine, the response has been twofold: to emphasize the importance of understanding basic patient values rather than speculating on future circumstances long in advance, and to conceive of the advance directive/surrogacy process as an evolving conversation over time in response to changing medical circumstances. ${ }^{10,11}$ This transformation has been helpfully summarized as a movement from a legal-transactional to a communications model. ${ }^{12} \mathrm{~A}$ parallel transformation must occur in the ethics of biobanking.

The responsibility to gain familiarity with donor values is complicated by biobanking's dual analogue to the clinical patient-both individual research recruits and target communities. Ideally, community engagement by the biobank addresses both, inviting individuals and communities into mutual dialogue on biobanking issues. Robust public engagement becomes akin to a surrogacy-advance directive conversation in responsible biobanking. ${ }^{13-15}$ (Although some biobanks already conduct admirable community engagement processes, the surrogacy model articulates the underlying rationale that is obscured by the consent model. It is not clear why community engagement would be necessary if individual informed consent to donate were coherent. Each recruit could just make his or her individual decision on risks/benefits, including on risks/ benefits to his or her community, and that would be that.)

As biobanks are ultimately making the concrete decision regarding which research to support, they have the responsibility to ascertain empirically their recruits' research goals and values. Donor values are relevant both to their protection from potential research harms and also to their interest in the social purpose of research in which they will participate. ${ }^{2}$ Important 
donor values may not be captured by specific categories of potential future research (the tiered consent approach) but might relate to broader goals: the aim to decrease race-related health disparities, or the desire to avoid complicity in abortion, etc. Conversely, general goals or values that are frequently stated by participants in public engagement within a specific community may suggest relevant categories or constraints to offer in specified options for individual donors.

The second responsibility of a surrogate is to be trustworthy to act on the values of the designee. In the clinical context, people may not trust all those who know them well. Family members or other intimate acquaintances may not share their ultimate values. So patients choose surrogates they trust not only to realize but also to carry out patient wishes, even when those are in tension with the surrogate's own values. Surrogate trustworthiness of a biobank is challenging to earn for several reasons. Unlike in clinical medicine, biobank recruits are not able to choose who will represent their interests in future decision making, although target communities may influence biobanks' ethical oversight structure. Like the clinical surrogate, the biobank must integrate considerations of donorautonomy ("what do weknowaboutdonor values?") and donor beneficence ("what is good for the donor at this unanticipated juncture?"). But integrations of autonomy and beneficence are additionally complex for biobanks because both individuals and communities are donors, incurrers of risk, and potential beneficiaries. Appropriate surrogacy thus demands conscientious discernment of community values, including areas of consensus and division, and complex decisions regarding policy implications of minority views. (Such decisions might include, for example, judgments about when to require recontact and specific consent for studies using de-identified samples.)

The biggest challenge to trustworthiness is biobanks' conflict of interest between their role as surrogate decision maker for donors and their other legitimate roles. It would be incoherent to ask of biobanks what is asked of clinical surrogates: to simply resist interests at odds with patient interest. As the purpose of biobanking is to facilitate research, not to directly benefit its donors, biobanks never make decisions solely in donors' best interests. Biobanks' overall mission is to increase knowledge that provides general social benefits. Conflicts may emerge between what kinds of research donors prioritize and what kinds the biobank considers most promising. Although biobank recruitment is local, research enterprises are global, so biobanks are under pressure for wider and wider sharing of samples as well as data for population studies. However, distinct local donor pools may have distinct relevant values or may prioritize local benefits from the research. In addition, financial motivations and associated conflicts of interest abound. Many biobanks are commercial enterprises whose existence is driven by fiduciary obligations to shareholders. Academic biobanks face financial pressures of grant competition. Even public health biobanks may be constituted with economic development goals as well as research goals in mind.

The intrinsic conflict of interests among legitimate biobanking goals results in a paradox. Biobanks de facto must be surrogate decision makers for their donors-they will make decisions on how banked samples will be used in research. Yet, ethically, biobanks should be precluded from being surrogates. That would be akin to having one's physician as one's surrogate decision maker in the clinical analogy-which of course is disallowed because constraining nonpatient-centered professional biases is one of the goals of clinical surrogacy.

Recognition of this paradox-that biobanks both must be and should not be donor surrogates-requires the development of governance structures that enable trustworthy representation of donor interests within the biobank. For an institution to be ethically constrained from some activities that otherwise support its mission is neither a new nor an insurmountable challenge. Many institutions support internal organizations designed to enforce such constraints: research institutions have institutional review boards, hospitals employ patient ombudsmen and form ethics committees, etc. Biobanking may be one of many emerging biotechnology institutions which require new models of "public interest governance."16

Similarly, progressive biobanks are beginning to include community advisory boards not only in public engagement but also in governance structure. At their best, these boards become the linchpin for two sets of checks and balances that bolster surrogate function: the community board's check, through governance oversight, on the research supported by the biobank and the donor's community check, through public engagement, on the community board's makeup and activities. (While questions of representation are inevitably challenging, these bodies are intended to mirror the diversity of the wider community. Membership procedures and guidelines could help to ensure accomplishment of that goal. For example, just as institutional review boards are required to have "community members" who are not connected to the research community, so too these bodies could have requirements that ensure voice for community members with no vested professional interest in research. Community feedback on membership can also be obtained.) In this way, biobanks can be explicit to donors about their commitment to their own interests while assuring that donor voices will be considered in ultimate biobanking decisions.

Finally, biobanks, like clinical surrogates, must acknowledge that the initial values and priorities of their trustees may change with differing circumstances and life stages. Unlike in the clinical model, the surrogate designees-both individual donors and their communities-remain competent and available for consultation over time. Community advisory boards can work to ensure an iterative circle between community engagement, donor consultation processes, biobank policy, and feedback to the community-for example, by providing an easily accessed and layperson-friendly public record of research proposals supported by the biobank. Research proposals that community engagement suggests may be controversial but that the biobank considers desirable could be flagged in such public records or in virtual systems connecting donors and biobanks - rather than relying on donors to monitor all possible studies. In this way, 
responsible surrogacy could enhance efforts to give more direct control to donors despite their temporal and physical detachment from research.

\section{INSIGHTS OF THE SURROGACY MODEL: CONCLUSION}

The strengths of the surrogacy model directly address multiple inadequacies of the informed consent model in biobanking. (i) The informed consent model presupposes known research goals with predictable potential benefits and risks. Alternatively, the surrogacy model facilitates decision-making about unknown future scenarios. (ii) Unlike the unidirectional information flow in informed consent in research, the surrogacy model stresses two-way dialogue between patient and the surrogate designee, clarifying patient values relevant to future unknowns. (iii) The informed consent model envisions a one-time consent process. The surrogacy model incorporates the evolution of patient values over time in response to changing circumstances over time.

Finally, a surrogacy model increases honesty to biobank recruits. Currently, those recruits are asked to make "informed" decisions about unknown future research with unknown risks and benefits-an impossible task. Instead of being placed in a position artificially likened to that of research consent, biobank donors should be told of the limits to donor choice, while being provided with a framework that assures them that their values and goals will be respected as much as possible. The surrogacy analogy helps to "flesh out" contemporary calls for biobanks to act as charitable trusts or as stewardships. ${ }^{17,18}$

By ignoring intrinsic incoherencies, ethical appeals that focus on obtaining informed consent from biobank recruits at the time of tissue donation pretend the key issue is choice rather than trust. But to have meaningful choice, biobank recruits and the communities in which they are located must have some surrogate embedded in biobank governance-a surrogate they trust to give permission for their tissue use or to recontact them when ethically required. And the surrogates must earn that trust.

\section{REGULATORY IMPLICATIONS: A CASE FOR MULTIPLE ANALOGIES}

Recognizing advantages of a surrogacy model does not negate the genuine ethical intuitions of the informed consent model. The research goals of biobanking pose the same ethical dangers for human subjects' use that exist in other forms of research, even if the decision-making by potential participants in biobanking is more akin to advance directives than to informed consent. Although the clinical surrogate's singular ethical duty is to make decisions on the patient's behalf, biobanks have other primary ethical duties consonant with their research mission. Therefore, whereas clinical surrogates must "bracket" potential conflicts of interest, biobanks must negotiate unavoidable conflicts of interest to avoid the overinstrumentalization of human research subjects.

Biobanking is a unique development in institutionalized research. Given that uniqueness, no one ethical analogy may be an adequate prism. "Thinking through" biobanking ethics through the lenses of multiple relevant, but imperfect, analogies may suggest the most robustly protective policies.

Significantly, current regulatory frameworks appeal only to one of the analogies we propose to integrate. Both present Department of Health and Human Services Office of Human Research Protection guidelines and advanced notice of proposed rule-making changes to the Common Rule 45 Cfr 46 define donation to a biobank as an act of research. The laudable intent of these regulations is to protect people from becoming research participants without their knowledge, by having tissue taken from them surreptitiously for research. But when that definition forces ethical conversation to rely solely on the framework of informed consent, attention both to the limits of donor autonomy and to donor beneficence may be diverted. Given the physical and temporal detachment of the donor from the research, "informed consent" paradoxically could become the legitimated means by which people are used in ways to which they might object or that are not in their best interest. It could become the means by which the burden of assembling evolving relevant information for risk/benefit analysis is transferred from research institutions to research recruits. Or informed consent might become an undue burden on genuine respect, for example, by forcing all biobanking to use an opt-in system when some kinds of research may be so widely supported that an opt-out system would be consonant with community values and individual choice.

Regulatory options that encourage the development of donor "advance directives" and surrogacy structures should also be explored. Ultimately, different strategies based on different analogies may be needed to ensure that donor interest acts as an ethical side constraint on biobanking activities.

\section{ACKNOWLEDGMENTS}

The authors gratefully acknowledge feedback on an oral presentation of this argument from attendees at the 2011 Exploring the ELSI Universe conference, 12-14 April 2011, at the University of North Carolina at Chapel Hill, Chapel Hill, NC. They also gratefully acknowledge the editorial assistance of Judith Andre and of two highly constructive anonymous peer reviewers.

\section{DISCLOSURE}

The authors declare no conflict of interest.

\section{REFERENCES}

1. Skloot R. The Immortal Life of Henrietta Lacks. Crown Publishers: NY, 2010

2. Tomlinson T. Protection of Non-Welfare Interests in the Research Uses of Archived Biological Samples," in K. Dierick and P. Borry, eds., New Challenges for Biobanks: Ethics, Law, and Governance. Antwerp, Belgium: Intersentia, 2009: 99-110.

3. US Department of Health \& Human Services. ANPRM for Revision to Common Rule. http://www.hhs.gov/ohrp/humansubjects/anprm2011 page.html

4. Hansonn MJ, Dillner J, Bartram CR, Carlson JA, Helgesson G. Should donors be allowed to give broad consent to future biobank research? Lancet Oncol 2006;7:266-269.

5. Maschke KJ. Alternative consent approaches for biobank research. Lancet Oncol 2006; 7:193-194.

6. Simon CM, L'heureux J, Murray JC, et al. Active choice but not too active: public perspectives on biobank consent models. Genet Med 2011;13: $821-831$ 
7. Trinidad SB, Fullerton SM, Ludman EJ, Jarvik GP, Larson EB, Burke W. Research ethics. Research practice and participant preferences: the growing gulf. Science 2011;331:287-288.

8. Allen J, McNamara B. Reconsidering the value of consent in biobank research. Bioethics 2011:25:155-166.

9. Sulmasy DP, Snyder L. Substituted interests and best judgments: an integrated model of surrogate decision making. JAMA 2010;304: 1946-1947.

10. Lambert P, Mclver Gibson J, Nathanson P. The Values History: An Innovation in Surrogate Decision-Making." J Law, Medicine, and Ethics 1990;18: 202-212.

11. Doukas DJ, Lindberg KA, Gorenflo D. An elderly population's medical values and advance health care directives. Fam Med 1994;26:610-611.

12. Sabatino CP. The evolution of health care advance planning law and policy. Milbank Q 2010:88:211-239.
13. Secko DM, Preto N, Niemeyer S, Burgess MM. Informed consent in biobank research: a deliberative approach to the debate. Soc Sci Med 2009;68: 781-789.

14. O'Doherty KC, Hawkins A. Structuring public engagement for effective input in policy development on human tissue biobanking. Public Health Genomics 2010;13:197-206.

15. O'Doherty KC, Burgess MM. Engaging the public on biobanks: outcomes of the BC biobank deliberation. Public Health Genomics 2009;12:203-215.

16. Caulfield T. Trusts, patents, and public perceptions: the governance of controversial biotechnology research. Nat Biotechnol 2006;24:1352-1354.

17. Winickoff $D E$, Winickoff RN. The charitable trust as model for genomic biobanks. N Eng/ J Med 2003;349:1180.

18. Williams PH, Schepp K, McGrath B, Mitchell P. The stewardship model: current viability for genetic biobank practice development. ANS Adv Nurs Sci 2010;33:E41-E49. 